\title{
Living in the epilepsy treatment gap in rural South India: A focused ethnography of women and problems associated with stigma
}

Jane R. von Gaudecker ${ }^{\mathrm{a}}$, Ann Gill Taylor ${ }^{\mathrm{b}}$, Arlene W. Keeling ${ }^{\mathrm{b}}$, Janice M. Buelow ${ }^{\mathrm{a}}$, and Sailas Benjamin $^{\mathrm{c}}$

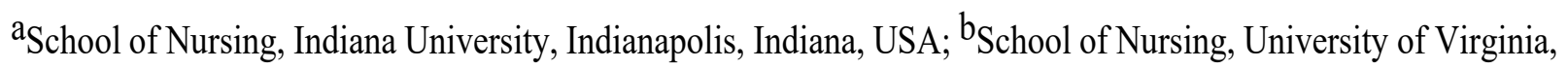
Charlottesville, Virginia, USA; ${ }^{\mathrm{C}}$ University of Calicut, Malappuram, India

\begin{abstract}
In India, women with epilepsy face unique challenges. A focused ethnography of six women within the epilepsy treatment gap was conducted in rural South India. Women were asked to describe their day-to-day lives. Data were collected through open-ended, semi structured interview questions, participant observation, and field notes. Thematic analysis was done. The disease-related stigma contributed to the women's physical, psychological, and emotional struggles; the women and their family members made every effort to conceal the disease.

Educational interventions to create awareness could help women seek effective treatments for their seizures, thereby reducing the stigma and improving the quality of their lives.
\end{abstract}

This is the author's manuscript of the article published in final edited form as: von Gaudecker, J. R., Taylor, A. G., Keeling, A. W., Buelow, J. M., \& Benjamin, S. (2017). Living in the epilepsy treatment gap in rural South India: A focused ethnography of women and problems associated with stigma. Health Care for Women International, 38(7), 753-764. https://doi.org/10.1080/07399332.2017.1321000 
Epilepsy, a chronic neurological disease characterized by recurrent seizures, is not only a medical diagnosis, but also a social label (Jacoby, Snape, \& Baker, 2005). Apart from the debilitating effects and unpredictability of seizures, persons with epilepsy (PWE) suffer from psychosocial burdens that can be worse than the disease itself, resulting in poor quality of life. In many cultures, the vulnerability of living with this disabling disease brings stigma, leading to discrimination and prejudices in many areas of life (De Boer, Mula, \& Sander, 2008). The challenges for PWE and their families increase when the disease is not treated appropriately, a phenomenon called the epilepsy treatment gap (ETG) (Meyer et al., 2012). By definition, the treatment gap is "the number of people with an illness, diseases, or disorder who need treatment but do not get it, expressed in percentage" (Kale, 2002, p. 31). The treatment gap for active epilepsy exceeds $75 \%$ in developing countries (Meyer, Dua, Ma, Saxena, \& Birbeck, 2010). In India, although the ETG in urban areas is $22 \%$, in rural areas, it is reported to be above $90 \%$ (Meyer et al.,2010; Santhosh, Sinha, \& Satishchandra, 2014). An improved understanding of how vulnerable population live with the disease, especially when it is not appropriately treated can help develop interventions to reduce treatment gap.

In spite of its low economic growth, the South Western coastal state of Kerala is heralded as the most developed state in India (Saradamma, Higginbotham, \& Nichter, 2000). Historically, the health status of women in this state has been considered superior compared to other parts of South Asia, although striking gender disparities remain (George, 2011; Thresia \& Mohindra, 2011). In Kerala, the prevalence of chronic and acute diseases is greater among women than men (George, 2011). Specifically, regarding epilepsy care, gender bias is seen in that fewer women with epilepsy (WWE), especially from lower socioeconomic status receive tertiary care in the state compared to their male counterparts. (Thomas, Deetha, Nair, \& Sarma, 2006). Furthermore, in a study conducted among 82 WWE living in Kerala, 55\% of these women reported concealing a history of the disease during marriage negotiation because of fear of breaking up the negotiations and eventually facing divorce, separations, and unhappy marriages (Santosh, Kumar, Sarma, \& Radhakrishnan, 2007).

While the above quantitative studies reported the experiences of women living with epilepsy, there is a lack in qualitative research that describes the day-to-day lives of 
women living within the ETG in Kerala. Understanding their lives is essential to guide culturally appropriate interventions and to improve the quality of life of WWE in the area. In this article, we identify and describe the struggles and aspirations of six women living within ETG in Kerala and suggest opportunities for future studies, including culturally appropriate interventions.

\section{Methods}

\section{Design}

To gain an in-depth understanding of the daily lives of WWE, living in the outskirts of Kozhikode district in Kerala, we conducted a qualitative descriptive focused ethnography. A focused ethnography is conducted in shorter duration than standard ethnography and with more intense data collection (Knoblauch, 2005).

\section{Data collection and analysis}

Prior to data collection, Human Subject Committee approval was obtained from institutional review boards of a major mid-Atlantic academic medical center and the Indian human ethical committee. Prior to conducting this study, the first author traveled to the proposed study area several times, connected with the traditional healers (those who prescribe Ayurvedic medicine, herbal remedies, and those who heal based on religious and indigenous beliefs with no professional medical training) to understand the various treatment options available for people with epilepsy. She reconnected with these healers during this study and to identify potential study participants. Data were collected from August to October 2014, through interviews, observations, and field notes. Interviews were conducted by the first author who visited each participant in their homes over a 2week period. The initial interviews were guided by 15 open-ended, semi structured questions. Because the women preferred staying at home, the majority of observations were based on their interactions with members of the family and their neighbors. These observations guided subsequent interviews and were used for triangulation purposes.

The first author who is native to Kozhikode district in Kerala lived in the area during data collection for the study and fluently spoke the local dialect (Malayalam), thus playing an insiders' role by immersing herself in the community. This in turn also led 
to developing a thick description for the study (Creswell \& Miller, 2000; Geertz, 1973). Data were collected from women $(N=6)$ who were medically diagnosed with epilepsy. The interview questions were open ended and allowed participants to talk freely; it was conducted in Malayalam and audio recorded (with consent). The interviews were transcribed verbatim and translated to English by the first author. The translated data were reviewed again with the audio recording to ensure accuracy of translation. For ease of management, data were then transferred into NVivo 10 software (NVivo, QSR International, version 10). Then, thematic analysis was conducted (Cohen, Kahn, \& Steeves, 2000). The data analysis was processed concurrently with data collection while exploring the meaning of what was said by the informants. Observational data were recorded daily as field notes and triangulated with interviews. Reviewing previous interviews and the field notes helped in developing follow-up questions. The process of data immersion was adopted through multiple readings to aid in coding, followed by data reduction. After these steps, data were subjected to line-by-line coding to identify commonalities, emerging patterns, and relationships (Cohen et al., 2000) to define categories. Overarching themes were identified through consensus from members of the research team during analysis.

\section{Sample}

WWE (key informants; $N=6$ ), family members taking care of these women (general informants; $N=8$ ), and traditional (indigenous) healers taking care of the participants (general informants; $N=2$ ) participated in the study. The six key informants were identified through the traditional healer's area of practice $(n=4)$ and through word of mouth $(n=2)$. Inclusion criteria for the key informants included being 18 years or older, a diagnosis of epilepsy from a qualified allopathic medical practitioner, receiving treatment under a traditional healer (e.g., ayurvedic, religious or indigenous based healers) for their seizures, and/or not adhering to the antiepilepsy drugs (AED) protocol. Women were excluded from participation if they had a diagnosis of nonepileptic spells, were pregnant or lactating, or were not mentally competent to participate.

\section{Key informants}


The six participants were between 20 and 63 years (mean=39.5 years) of age and lived in different locations on the outskirt of Kozhikode district. Three participants were married; one was single, one widowed, and one divorced. One participant had a master's degree, two participants had high school education, two participants had elementary school education, and one participant was illiterate. One participant worked as an office clerk, one was a daily wage laborer, and the other four participants were unemployed. These participants had a medical diagnosis of epilepsy ranging from 4 to 42 years (mean $=21.5$ years).

\section{Results}

Initially, 21 women were identified as potential participants. Among them, eight were young in their marriage age and therefore refused to allow the first author to visit their home for interviews or participate in this "epilepsy" study for fear of disclosure of their disease condition to their neighbors that may have subsequent negative effects on marriage negotiations (von Gaudecker, 2015). All women who eventually participated in the study referred to the first author (interviewer) as their friend or distant relative when asked by curious neighbors who the visiting person was. In compliance with the participants' requests, all the interviews and daily visits for this study were conducted at their homes.

Six interrelated themes emerged from the data, with three of these revealing some of the major struggles and aspirations shared by the WWE. These themes were (a) "scarring and stigmatization," (b) "have to suffer," and (c) "live my life." The general belief among the participants and their families was that epilepsy was caused by a divine curse, an evil spirit, and/ or being possessed. These beliefs guided them in seeking different types of treatments. Four of the women who were on antiepileptic drugs were taking less than the prescribed dose, and two other participants were on self-treatment or no treatment at all. Three additional themes that emerged are regarding participants' decisions about treatment choices and barriers to effective care.

\section{Theme 1: Scarring and stigmatization}

Participants in this study shared the many struggles they undergo when living with this disease. Epilepsy caused "outer" scarring caused by the physical injuries acquired as a result of the seizures and "inner" scarring related to the psychological and emotional 
struggles of living with the disease. All the participants reported having seizures that caused fall-related injuries such as fractures, burns, cuts, and bruises. They also reported that the seizures were occasionally associated with fecal and/or urinary incontinence and exposure of their body parts, which caused them much shame. One participant who was employed as a clerk in a local company reported that after such an incident of body exposure during a seizure at her work- place, she resigned from her job.

The impact of the disease goes much beyond physical injuries. The participants and their families took every effort to hide the diagnosis of epilepsy from the community, distant family, and friends, indicating the severe burden of stigma related to the disease. One participant who had nocturnal seizures reported that her husband's family, who lived in the same house with her, was unaware of her seizures. In an effort to conceal the disease and to avoid the embarrassment of having a seizure in public, she limited her social inter- action much like all study participants. Most of these women remained at home, avoiding even family unions and ceremonies such as weddings. This limitation in socializing and the resulting frustration was echoed in their responses. One participant stated "They [her mother, brothers and two daughters] tell me not to go anywhere... I do not go for any work, I cannot go for any function [gatherings]. And even if I go, I come back quickly..." Thus, the disease also had an impact on family members as they restricted participants from engaging in social activities for fear that they would have a seizure in the presence of others. Another participant echoed similar sentiments "I can- not go anywhere with this [seizures]... I do not have the courage to go alone. No one knows when it [seizures] will happen..." Yet another participant reported that she avoids visiting even her neighbor.

Living with epilepsy was burdensome not only for the participants, but also for their families. The concerns and uncertainty about the women's future reverberated in their words. A mother of one participant said: "As long as I live, I will take care of her. Who will be there for her after I am gone?"

Another participant shared:

Because of this [seizures], my mother often says: 'I wish you'd die before I do.' That is hurtful [to hear]. Who will be there for my children? She [mother] worries that if she dies, no one will be there for me... 
These statements from the family members clearly created a sense of confusion and a feeling of burden that the women are imposing on others; this deepened the "inner" scars caused by the disease.

Four of the young participants had unique struggles in relation to their married life. Arranged marriages, where elders in the family find appropriate matches for their children who are of marriage age, are customary in this community. These young women and their family members concealed the young women's history of epilepsy during the marriage negotiations for fear of breaking up the marriage proposal. Two of the women reported that it was their family's decision to conceal the history of the disease, even though the participants themselves were not in favor of hiding the disease. One woman who had a master's degree had also concealed the history of her disease from her fiance. After her marriage, the disease was accidently disclosed when her husband inquired at the pharmacy about the medication she was taking, later witnessing one of her seizures. The following excerpt reveals the struggle the participant now faces from her spouse:

His [husband's] anger is shown on me.... when he is upset, he says, "you have this dis- ease, your family cheated me [so now], whatever I do, you have to adjust accordingly and live." ... [Meaning], even if he is wrong, or makes any mistake, I have to adjust because I have this disease.... sometimes he sits and cries, saying he was cheated and blames his own fate.... He has also started drinking [alcohol] more and comes home very late.... I did not bring this disease on me...

Concealing the disease from their spouses eventually created a sense of guilt and

regret among the young married participants. One participant said:

I feel bad for him [husband]. If he had not married me, he could have gone many places. He loves travelling. I do not have the courage [to go out] ... It's [seizures] a burden for others... I wish I did not get married... I think of my husband and wish so. Isn't it sad for him? He would have had a lot of dreams and it's all gone because he married me.

Another participant who was emotional during conversations regarding her married life stated:

$[\mathrm{He}]$ is very angry with my family [for concealing my disease] and often shows it on me... he [husband] tells me that my family cheated him.... Most of his relatives live far [from us]. I don't go there because I don't stay anywhere overnight... I don't want anyone to know [about the seizures] ... He tells me that because of me he is not able to visit his relatives... What I really am regretting now is that I am married. I wish I was not. Even if someone physically hurts me, that's fine. Why can't he understand this [the disease] is not my mistake?

\section{Theme 2: "Have to suffer"}


Most of the women accepted the struggles they faced as something they had to live with.

However, the struggles caused them much suffering. All participants considered the disease as their fate. One participant said:

Everything happens according to our fate. It [seizure] doesn't happen every day. Let everything happen according to my fate... its God's will. I say this and comfort myself... I have gone to many [healers]. They all say it is a difficult disease to find cure. We just have to suffer.

All six participants echoed similar sentiments about living a seizure free life.

Because of the history of this participant's epilepsy, her daughter's marriage was delayed.

The participant attributed this to her fate. Another participant commented: "This

[seizure] does not happen every day... We have to live with what God has allowed in our

life..." All the participants had the same attitude about their disease. In their despair, they

reported that they often questioned the supreme power [God] on why they were chosen to

undergo these struggles.

The disease, according to the participants, was something they had to deal with

quietly. Despite the struggles they face, five out of six women reported that they had never spoken with anyone about their struggles with the disease. One participant commented:

This [seizures] is a burden for them [family], why should I worry them even more [by talking about it]? I have severe headache and heaviness of head after seizure and I try not to trouble them [family] even by saying that....

Considering herself as a burden to her family, she discontinued her studies for which the family had to pay, and skipped taking medicines to make her antiepileptic drugs last longer, thus saving money for her family. Yet another participant who had concealed the history of her disease and struggled with her husband's attitude toward her because of the disease stated:

He knows that I will not tell anyone about it [he being upset with her], because if I do, I will have to tell them about my seizures as well and I don't want that... We did not tell him before marriage [about seizures] and what right do I have to say anything now? I just have to silently face this.

One of these participants who occasionally spoke with her friend about her struggles with seizures noted that she often found relief in doing so.

\section{Theme 3: "Live my life"}

Like most healthy individuals, these women had dreams and future aspirations. When asked about them, the participants were quick and spontaneous to narrate. Finding 
happiness and living their lives in spite of the chronic disease was a hope for all six of the women. Being independent, leading a happy married life, giving birth to "normal" children, and avoiding side effects of medications were the most common responses. One participant stated: "I wish I could work... I wish I could attend weddings and other functions... if I didn't have this (seizures), I could have done everything, and I could have lived my life...." Another participant who was preparing for her marriage said:

I dream of leading a happy family life with my husband in his house ... without troubling them [his family] in any way... [Sometimes] when a daughter-in-law goes to her husband's house, we hear a lot about the daily fights and quarrels... But without any of that...

She had concealed her diagnosis from her fiance and was optimistic that she can hide the medications and was hopeful that they will not discover her diagnosis. Culturally, a woman who lives in rural Kerala moves to husband's house where his parents and other family members also most often live. Her concern was that if her disease would be disclosed, there would be constant quarrels between her and her in-laws.

Apart from the struggles of living with the disease, the participants who were on AEDs had side effects of these drugs, although they did not take the prescribed dose. They considered their problems two pronged - the disease itself and the side effects from the treatment. One participant said: “... [I wish a life] without [listening to] any hurtful words [from husband] and without allopathic [AEDs] medicines. I do not like the side effects of those medicines... and I wish my husband will understand me and my sickness..."

However, the participant had never dis- cussed about the side effects of the AEDs with her physician but sought treatment to relieve those through traditional healers.

All the young participants had concerns about their offspring. One participant who was married a year ago had had a miscarriage a few months prior to this study and was especially concerned about giving birth to a normal child. She was concerned that her miscarriage was related to her seizures and the medicines she taking. Like all the young participants in this study, her wish for future was to give birth to healthy children and make her husband happy.

\section{Discussion}

The purpose of this focused ethnography was to describe the lives of women living within the ETG in rural Kerala, South India. The authors bracketed all prior assumptions about 
what the women's life might be like and immersed themselves in the data the women provided. Our attempt was to hear the participant's stories and understand how they live day to day with this chronic disease, and how they make meaning of their experiences. The goal was to understand the experiences of women living with epilepsy by providing a window through which to view their everyday lives and a foundational understanding of the struggles and the aspirations of these women.

Historically, epilepsy has been a stigmatized disease (Jacoby, 2002). The overall study findings suggest that epilepsy continues to be a stigmatizing disease in this community because of the prevailing myths and misconceptions. Stigma was evident during the recruitment process because young women of marital age refused to participate in this study. Living in a patriarchal society such as in rural India, women are subordinate to men and older relatives in the family (Coffey, Spears, \& Khera, 2013). For the women living in Kerala concealment of their disease was their response to the existing stigma related to seizures.

One of the major reasons for this stigma is the limited knowledge and false beliefs about epilepsy that exist in this community. The attitude of family members also suggested that they contributed to the existing stigma by taking every effort to conceal the participants' disease and/or their hurtful behaviors toward the participants. In a door-to-door survey conducted earlier in Kerala, $68 \%$ of the respondents believed that epilepsy was a brain disease (Radhakrishnan et al., 2000). However, despite the high literacy of the people of Kerala, the six participants who were living within the ETG believed myths and had misconceptions that were deep rooted and contributed to the stigma they felt. This could also suggest that these false beliefs led to the existing treatment gap among these participants. Although assessing the community's attitude toward people with epilepsy was beyond the scope of this study, the reason for the participants to conceal their disease from the community was because the participants feared the existing stigma. This suggests possible gaps in the understanding of the disease by the public. The concept of enacted stigma among poorly informed public is well documented in the Western world (Kılınç\& Campbell, 2009; Scambler \& Hopkins, 1986). 
The struggles of any woman living with a chronic stigmatized disease can be devastating. From the participant's responses, it is evident that the psychosocial consequences of living with epilepsy caused more distress than the seizures. The fear of how these participants will be accepted with such a diagnosis and the possible negative consequences amplify the perceived stigma about the disease. Although the participants concealed their epilepsy from prospective spouses with the intention of promoting a happy marriage, the women who did get married were eventually unhappy, felt guilty, and experienced marital difficulties. Our findings suggest that women of marriage age are more vulnerable and stigmatized in this society, supporting the findings of the study conducted earlier in Kerala (Santosh et al., 2007).

Epilepsy is especially a debilitating disease, not just physically but psychologically and emotionally. The participants were worried about the uncertainty of the disease and the embarrassment and shame of having a seizure in the presence of others. More than the physical injuries, the possibility of incontinence and body exposure worried them. The consequences of living with the disease are worse when it is not treated adequately and seizures are not well controlled. A logical first step in reducing the burden of living with epilepsy for these women is to educate them about the scientific cause of epilepsy and to facilitate them in achieving seizure control. Seizure reduction can considerably improve the quality of their life (Birbeck, Hays, Cui, \& Vickrey, 2002). Although there is no cure for epilepsy with pharmacotherapy, $70 \%$ of the time, AEDs can help in controlling seizures (Schmidt \& Schachter, 2014). The women in this study lived within the ETG, which can explain the reason for their struggles. Promoting a clear understanding of the cause for epilepsy and the appropriate ways to effectively treat the disease could reduce the stigma associated with epilepsy and encourage WWE to continue with their treatment. It is important that these women and their families conceptualize epilepsy as a neurological disease and that the disease is different from who the person is (Aujoulat, Marcolongo, Bonadiman, \& Deccache, 2008).

\section{Limitations}

The participants were recruited through purposive and snowball sampling, and therefore, possibility of selection bias cannot be denied. It is possible that a certain subset of women 
living within the ETG who were willing to talk about their disease participated in the research. All the young participants were identified through traditional healer's consultation area; women's participation in the study was based on their trust on the healers and their influence, again suggesting a possibility for selection bias.

Our attempt has been to narrate the stories of these women and make their voices heard. We have neither generalized the findings of this study nor singled out Kozhikode district as the only place where challenges of living with epilepsy exist. We have provided a close personal look at how women within ETG live in this community. India is the second most populous country in the world with a diverse culture. The experiences of these participants may not be similar to the experience of WWE living elsewhere in rural or urban India.

\section{Recommendations}

Educating affected individuals and the community at large about the scientific cause of epilepsy is the most important first step toward reducing the ETG and improving the quality of life for women with living epilepsy in India. Such educational intervention should be culturally based by acknowledging the practices and cultures of the given community. Women in this study trusted their healers. There- fore, educating traditional healers (e.g., indigenous and religious) and grass-root level community health workers about the scientific cause of epilepsy and promoting awareness and education through them to the community could result in an acceptable and positive outcomes.

While we do not make any attempt to generalize the findings of this study, it has the potential to guide future researchers to develop studies, and to plan culturally appropriate interventions toward reducing stigma and treatment gap. We suggest that further studies be conducted to describe the difficulties of living with epilepsy, to make gender-based comparison between people with well-controlled epilepsy and those within the ETG in both urban and rural parts of India.

\section{Conclusions}

Through intense field immersion, triangulation of interview, and observational data, this study is the first of its kind in India to shed light on the day-to-day experiences of living with 
epilepsy in rural India and to suggest possible reasons for the ETG among these participants. The ignorance and false beliefs about the cause of epilepsy likely contribute to the stigma and add stressors for the participants and their families. These struggles are perceived to be more burdensome than the disease itself.

\section{Acknowledgments}

Appreciation is given to Dr. V.M Kannan, at the University of Calicut, for his guidance with the local ethical board approval of the protocol for this study. Special thanks are given to all the six participants and their family members who participated in this study.

\section{Funding}

The first author acknowledges the funding received from Skinner Scholarship and the Global Health Scholar award program, Center for Global Health, University of Virginia. This publication was made possible by grant number 2T32 NR007066 from the National Institute of Nursing Research. 


\section{References}

Aujoulat, I., Marcolongo, R., Bonadiman, L., \& Deccache, A. (2008). Reconsidering patient empowerment in chronic illness: A critique of models of self-efficacy and bodily control. Social Science \& Medicine, 66(5), 1228-1239. https://doi.org/10.1016/j.socscimed.2007.11.034

Birbeck, G. L., Hays, R. D., Cui, X., \& Vickrey, B. G. (2002). Seizure reduction and quality of life improvements in people with epilepsy. Epilepsia, 43(5), 535-538. https://doi.org/10.1046/ j.1528-1157.2002.32201.x

Coffey, D., Spears, D., \& Khera, R. (2013). Women's status and children's height in India: Evi- dence from joint rural households. Unpublished report. Princeton, NJ: Office of Population Research, Wallace Hall, Princeton University.

Cohen, M. Z., Kahn, D. L., \& Steeves, R. H. (2000). Hermeneutic phenomenological research: A practical guide for nurse researchers. Thousand Oaks, CA: Sage.

Creswell, J. W., \& Miller, D. L. (2000). Determining validity in qualitative inquiry. Theory Into Practice, 39(3), 124-130.

De Boer, H. M., Mula, M., \& Sander, J. W. (2008). The global burden and stigma of epilepsy. Epilepsy \& Behavior, 12(4), 540-546.

Geertz, C. (1973). The interpretation of cultures. New York, NY: Basic Books.

George, M. (2011). In the midst of a storm: Distress of Kerala women. Affilia: Journal of Women \& Social Work, 26(3), 304-313. https://doi.org/10.1177/0886109911417690

Jacoby, A. (2002). Stigma, epilepsy, and quality of life. Epilepsy \& Behavior, 3(6, Suppl. 2), 10- 20. https://doi.org/10.1016/S1525-5050(02)00545-0

Jacoby, A., Snape, D., \& Baker, G. A. (2005). Epilepsy and social identity: The stigma of a chronic neurological disorder. The Lancet Neurology, 4(3), 171-178.

Kale, R. (2002). The treatment gap. Epilepsia, 43(Suppl. 6), 31-33. https://doi.org/10.1046/ j.1528-1157.43.s.6.13.x 
Kılınç, S., \& Campbell, C. (2009). "It shouldn't be something that's evil, it should be talked about": A phenomenological approach to epilepsy and stigma. Seizure, 18(10), 665-671. https://doi.org/10.1016/j.seizure.2009.09.001

Knoblauch, H. (2005). Focused ethnography. Forum Qualitative Sozialforschung / Forum: Qualitative Social Research, 6(3). Retrieved from http://www.qualitativeresearch.net/index.php/fqs/article/view/20

Meyer, A. L., Dua, T., Boscardin, W. J., Escarce, J. J., Saxena, S., \& Birbeck, G. L. (2012). Critical determinants of the epilepsy treatment gap: A cross-national analysis in resource-limited set- tings. Epilepsia, 53(12), 2178-2185.

https://doi.org/10.1111/epi.12002

Meyer, A., Dua, T., Ma, J., Saxena, S., \& Birbeck, G. (2010). Global disparities in the epilepsy treatment gap: A systematic review. Bulletin of the World Health Organization, $88(4), 260-266$.

Radhakrishnan, K., Pandian, J. D., Santhoshkumar, T., Thomas, S. V., Deetha, T. D., Sarma, P. S., \& Mohamed, E. (2000). Prevalence, knowledge, attitude, and practice of epilepsy in Ker- ala, South India. Epilepsia, 41(8), 1027-1035. https://doi.org/10.1111/j.1528-1157.2000. tb00289.x

Santosh, D., Kumar, T. S., Sarma, P. S., \& Radhakrishnan, K. (2007). Women with onset of epilepsy prior to marriage: disclose or conceal? Epilepsia, 48(5), 1007-1010.

Santhosh, N. S., Sinha, S., \& Satishchandra, P. (2014). Epilepsy: Indian perspective. Annals of Indian Academy of Neurology, 17(Suppl. 1), S3.

Saradamma, R. D., Higginbotham, N., \& Nichter, M. (2000). Social factors influencing the acquisition of antibiotics without prescription in Kerala State, south India. Social Science \& Medicine, 50(6), 891-903.

Scambler, G., \& Hopkins, A. (1986). Being epileptic: Coming to terms with stigma. Sociology of Health \& Illness, 8(1), 26-43.

Schmidt, D., \& Schachter, S. C. (2014). Drug treatment of epilepsy in adults. British Medical Journal, 348(254), 130-136. https://doi.org/10.1136/bmj.g254 
Thomas, S. V., Deetha, T. D., Nair, P., \& Sarma, S. P. (2006). Fewer women receive tertiary care for epilepsy in Kerala State, India. Epileptic Disorders: International Epilepsy Journal With Videotape, 8(3), 184-189.

Thresia, C. U., \& Mohindra, K. S. (2011). Gender bias in health research: Implications for women's health in Kerala (India) and Sri Lanka. Critical Public Health, 21(3), 327337. https:// doi.org/10.1080/09581596.2010.493172

von Gaudecker, J. R. (2015). Marriage and epilepsy: Struggles of women living with epilepsy in Kerala, South India. Epigraph, 17(3). 